\title{
FRANCISCO ANTONIO ZEA: PERIODISTA, BOTÁNICO Y POLÍTICO
}

\author{
Diana E. Soto Arango \\ Dpto. de H. ${ }^{a}$ de la Ciencia - Centro de Estudios Históricos, CSIC - C/ Duque de Medinaceli 6, Madrid
}

\section{RESUMEN}

Este trabajo analiza la faceta periodística de Francisco Antonio Zea, cuyo primer artículo, «Avisos de Hebephilo», se publicó en 1791 en el Papel Periódico de la ciudad de Santa Fé de Bogota. Su interés por la prensa se manifiesta en su etapa de estudiante en el Instituto Nacional de Francia, pero no será hasta 1803, a su regreso a Madrid, cuando evidencie sus dotes para la organización y dirección periodísticas en El Mercurio y en la Gaceta de Madrid. Posteriormente, siendo director del Real Jardín Botánico de Madrid, comparte la dirección del Semanario de Agricultura y Artes, y, finalmente, en 1818 funda y dirige el venezolano Correo del Orinoco.

\section{SUMMARY}

This work analyzes the journalistic facet of Francisco Antonio Zea, whose first article, «Avisos de Hebephilo», was published in 1791 in the Papel Periódico de la ciudad de Santa Fé de Bogotá. His interest for the press shows at his stage of student in the National Institute of France, but won't be up to 1803 , at his return to Madrid, when he proves his talent for the organization and journalistic administration on The Mercury and on the Gazette de Madrid. Later on, being manager of the Royal Botanical Garden of Madrid, he shares the management of the Semanario de Agricultura y Artes, and, finally, in 1818 establishes and directs the Venezuelan Correo del Orinoco.

\section{INTRODUCCIÓN}

En este trabajo nos ocuparemos de Francisco Antonio Zea en su faceta de periodista. Su primera publicación la realizó en Papel Periódico con el artículo «Avisos de Hebephilo». En éste criticaba el deplorable estado en que se encontraba la enseñanza superior en la capital del Virreinato.

No deja de ser importante su vinculación con los periódicos y revistas parisienses durante su época de estudiante en el Instituto Nacional de Francia. Pero donde demuestra su capacidad de organización y dirección periodística fue, precisamente, en El Mercurio y en la Gaceta de Madrid. Posteriormente, y siendo director del Real Jardín Botánico de Madrid, comparte la dirección del Semanario de Agricultura y 
Artes con los profesores del Jardín Botánico. En la época de su actividad política, de luchador por la independencia americana, tiene la oportunidad de fundar y dirigir el Correo del Orinoco en la ciudad de Angosturas, Venezuela.

\title{
AVISOS DE HEBEPHILO ARTÍCULO CRÍTICO DE LA EDUCACION NEOGRANADINA
}

\begin{abstract}
«Yo por lo menos siento los ardores de una llama que me incita a exponer a vuestros ojos el cuadro filosófico de nuestra ignorancia y de nuestras miserias, originadas por la mayor parte de esos estudios abstractos y científicas boberas, que sólo sirven para formar ciudadanos inútiles que la Sociedad mantiene a su pesar y para conservar como un depósito preciosos la barbarie y la rusticidad»! .
\end{abstract}

Hacia finales del Siglo XVIII el ambiente estudiantil de los colegios de Santa Fe tomó un rumbo diferente. Los estudiantes, antes sumisos, ahora reclamaban y protestaban; se quejaban de los estudios que se les impartían y, especialmente, de la filosofía peripatética que, como decía el fiscal Moreno en los años anteriores, antes que ilustrarlos «los embrutecía»².

Quizá el año de 1791 sea de mayor número de polémicas en los claustros colegiales. En este año, Francisco Antonio Zea ${ }^{3}$ sale a la palestra periodística con el Artículo «Avisos de Hebephilo» que editó en Papel Periódico y que correspondía a la dinámica de discusión que, en ese momento, se estaba dando en los Colegios Mayores del Rosario y de San Bartolomé.

1 ZEA, F.A. (1791), «Avisos de Hebephilo... o discurso previo a la juventud», en Papel Periódico de la ciudad de Santa Fe de Bogotá, números 8 y 9 de abril. Ha sido publicado en Hernández de Alba, G. (1983), Documentos para la historia de la educación en Colombia, Editorial Kelly, Bogotá, t.V, pp.203-213.

2 «Plan de estudios de Moreno y Escandón. Santafé, septiembre de 1774». Archivo General de Indias (en adelante AGI), sección quinta, Audiencia de Santa Fe, legajo 757.

3 Zea nació en la villa de Medellín, el 23 de noviembre de 1766 y murió el 28 de noviembre de 1822 en el York House Hotel, de la ciudad de Bath, Inglaterra, a la edad de 51 años. Zea tuvo a su favor el ser hijo de nobles, hecho que le permitió ingresar como estudiante del Colegio de Popayán y colegial en el Mayor de San Bartolomé de Santa Fe de Bogotá. Fue discípulo de José Félix Restrepo y compañero con Caldas y Torres. Zea empieza su vida profesional en 1788, como catedrático de gramática en el Colegio de San Bartolomé y en 1791 el virrey Ezpeleta le encargó la educación de sus hijos y en este mismo año se le nombra, por petición de Mutis, subdirector de la Real Expedición Botánica de la Nueva Granada. Participó en la tertulia «El Arcano de la Filantropía», que coordinaba Nariño y que, luego, fuera el motivo principal para su detención en 1795. 


\section{FRANCISCO ANTONIO ZEA: PERIODISTICA, BOTÁNICO Y POLÍTICO}

En el Colegio Mayor del Rosario, el joven profesor Manuel Santiago Vallecilla ${ }^{4}$, educado bajo el método del fiscal Moreno y Escandón, se enfrentaba al rector de su colegio centrando la discusión en la importancia del estudio de las matemáticas basadas en el método ecléctico, en las teorías de Newton y en las heliocéntricas. Vallecilla, de esta manera, se contraponía al espíritu de partido y al «método pernicioso de la escolástica» ${ }^{5}$.

Por su parte, los estudiantes del Colegio de San Bartolomé, dirigieron un escrito al Virrey solicitando que se les concediese poner a sus expensas un catedrático que les enseñara física, matemáticas, botánica e historia natural ${ }^{6}$. Los estudiantes bartolinos señalaban los dos objetivos que defendía el profesor Zea en sus escritos de «Avisos de Hebephilo» ${ }^{7}$. Estos eran: la enseñanza de la buena filosofía, basada en Newton, y que les hiciesen conocer el suelo que habitaban con las riquezas que les rodeaban.

En cuanto a las teorías de Newton, éstas se habían enseñado en el Colegio de San Bartolomé desde la época de los jesuitas y, luego, José Celestino Mutis las había introducido en su clases de matemáticas en el Colegio del Rosario ${ }^{8}$. Es decir, las teorías newtonianas no eran una novedad en el ambiente académico de Santa Fe. Sin embargo, no se habían podido institucionalizar por la contrarreforma del Plan de 1779 y la oposición de los sectores más tradicionales. De ahí que Zea, Vallecilla, y los estudiantes bartolinos abogaran por esta enseñanza en concreto.

La forma de solicitar la nueva enseñanza era diferente entre los estudiantes bartolinos y rosaristas. Así lo manifestaban los alumnos del Colegio de San Bartolomé cuando exponen que ellos utilizaban los medios que sugerían «la prudencia y la honestidad», en tanto que sus compañeros rosaristas «llegaban a zaherir y burlar a su rector a quien juzgaban celoso partidario de la filosofía peripatética» ${ }^{9}$.

Estas discusiones debían seguirse entre la intelectualidad criolla santafereña. Zea, en su escrito, demuestra un gran conocimiento de la intelectualidad del momento.

4 Véase un completo análisis de la polémica de Vallecilla en Soto ARAngo, D. (1993), Polémicas universitarias en Santa Fe de Bogotá. Siglo XVIII, Colciencias, Bogotá, pp.183-184.

5 Archivo Histórico Nacional de Colombia, (en adelante AHNC), sección Colonia, fondo miscelánea, t.31, f.54 v.

6 AHNC, sección colonia, fondo milicias y marina, t.128, fols.200-201.

7 Un análisis completo de este documento se localiza en ARBOLEDA, L.C. (1990), «La ciencia y el ideal de ascenso social de los criollos en el virreinato de la Nueva Granada», en Ciencia, Técnica y Estado en la España Ilustrada, Ministerio de Educación y Ciencia, Sociedad Española de Historia de las Ciencias y de las Técnicas, Zaragoza, pp.193-225.

8 Véase un análisis de estos estudios en ARboleda, L.C. y Soto Arango, D. (1991), «Las teorías de Copérnico y Newton en los estudios superiores del virreinato de la Nueva Granada y en la Audiencia de Caracas. Siglo XVIII», Revista Quipu, vol.8, n I, enero-abril, pp.5-34.

9 AHNC, sección colonia, fondo milicias y marina, t. 128, fols.200-201. 


\section{DIANA E. SOTO ARANGO}

Destaca a los maestros Restrepo, Valenzuela, Mutis y Moreno como los abanderados en el virreinato de la nueva filosofía ilustrada. Advertía, que se encontraban en una época «en que todo conspira a la ilustración de la juventud». Sin embargo, el ejemplo estaba dado y debía fomentarse «la ciencia del Newton y del divino Malebranche» 10 .

Utilizando el lenguaje moderado de los bartolinos y sabiendo que el virrey Ezpeleta no apoyaba al profesor Vallecilla, en su polémica contra el rector escolástico del Colegio del Rosario, pasa por alto esta actitud porque, según él, era «un Virrey superior a las preocupaciones escolásticas, lleno de las luces del inmortal Buffon, amante y cultivador de las bellas artes; $\ldots$ cuyos proyectos solo van dirigidos a conservar a la República de las letras, lo que el furor del peripato ha perdonado» ${ }^{11}$.

Es posible que Zea, en esas circunstancias, compartiera la actitud de moderación de Mutis ante las «revueltas» del Colegio del Rosario y entendiera la postura de su virrey de no apoyar al catedrático Vallecilla ${ }^{12}$. Se imponía en aquel momento el miedo a la «subversión», si se originaba desde los claustros universitarios. Pero, en cambio, fuera de éstos, el virrey apoyaba a la Expedición Botánica y a Zea mismo, al nombrarle unos meses después, a petición de Mutis, «Segundo Agregado de la Expedición Botánica de Santa Fe» ${ }^{13}$.

En su primer documento público, Zea insitió en criticar los estudios inútiles basados en la filosofía escolástica, que no solo formaba «orgullosos ignorantes», sino que, también, y lo más grave, que «deshonraba la humanidad, sometiendo los entendimientos a una ignominiosa esclavitud y servidumbre filosófica» ${ }^{14}$. Los objetivos de «sustituir las útiles ciencias exactas en lugar de las meramente especulativas» ${ }^{15} \mathrm{y}$ «hacer gustar los conocimientos útiles aunque sea de un modo elemental y diminuto» ${ }^{16}$, se venían planteando desde los primeros documentos de Mutis y siguieron

10) ZEA, F.A. (1791), p.208.

11 El virrey Ezpeleta gobernó el virreinato de 1789 a 1797. Ibidem.

12 Sin embargo, Zea apoya con su escrito a Vallecilla cuando plantea que: «finalmente un gran número de eruditos patriotas, empeñados en llevar sus luces, a las tenebrosas escuelas, en donde habita la ignorancia». Ibidem.

13 El virrey Ezpeleta nombra a Zea el 11 de noviembre de 1791. Véanse Botero Saldarriaga, R. (1945), Francisco Antonio Zea, Imp. Municipal, Ediciones del Consejo, Bogotá, p.38; OrTIZ, S.E. (1966), Colección de documentos para la Historia de Colombia. Epoca de la Independencia, Editorial ABC, Bogotá, p. 67.

14 ZEA, F.A. (179I), pp.204-205

1.5 Véase en el informe de mando del virrey Caballero y Góngora, en el Capítulo III, de la Instrucción Pública. J.M. PÉrez AYAlA, (1951): Antonio Caballero y Góngora, virrey arzobispo de Santa Fe, I723-1796, Imp. Municipal, Bogotá, p.341.

16 «Plan de estudios de Filosofía, para el Colegio de San Pedro Apóstol de Mompox, elaborado por Eloy Valenzuela», 2 de septiembre de 1806. Biblioteca Nacional de Colombia, (en adelante BNC), sección Libros Raros y Curiosos, Protocolo de Instrucción Pública, 1672-1818. Número 338, fls. 298- 


\section{FRANCISCO ANTONIO ZEA: PERIODISTICA, BOTÁNICO Y POLÍTICO}

señalándose como objetivos en la educación colonial y, posteriormente, en la naciente República de la Gran Colombia.

Debemos resaltar una constante en los escritos de Zea, que se manifestó desde este primer artículo: no sólo criticar, en este caso «la filosofía escolástica» y los «estudios inútiles», sino también caracterizar la situación del Virreinato y dar respuesta a los problemas planteados.

Para el profesor Zea su Virreinato «estaba sumergido en la última barbarie y, a pesar de su vasta extensión territorial, habitado solamente de un millón y medio de hombres miserables, sin ciencias, ni artes, ni agricultura, ni comercio en medio de su miseria, era el favorito de la naturaleza» 17 .

Las soluciones las planteó desde el diagnóstico de la realidad del Virreinato y teniendo en cuenta los recursos naturales que existían en el reino. Por otra parte, teniendo como punto de partida los recursos humanos, señalaba que se contaba con un «crecido número de genios» y se debía formar en éstos «el espíritu patriótico fundamentado en una educación literaria, política y civil» ${ }^{18}$, tomando conciencia de los intereses de la República.

\section{LA GACETA Y EL MERCURIO, MEDIOS DE EXPRESIÓN DEL GRUPO AFRANCESADO}

«Por fortuna ha tenido Francia un Bonaparte, sin el qual los partidos y la irreligión habrían devorado el fruto de tantas victorias: no habría conseguido la paz ni llegado al alto punto de engrandecimiento en que se halla»'19.

Zea quedó marcado, en lo científico y en lo político, por su estancia en París. En la capital gala establece relación con los directores de los periódicos y realiza pequeñas reseñas sobre los botánicos españoles donde él también se incluía ${ }^{20}$. A su regreso a Madrid, ya como segundo profesor del Jardín Botánico y, en especial, como direc-

309. Publicado en Soto, D. (1994), Las universidades y colegios mayores de Santa Fe, Quito y Caracas. Estudio Bibliográfico y de Fuentes, Colciencias/Universidad Pedagógica Nacional, Bogotá, pp.223-239.

17 ZEA, F.A. (1791), p.211.

18 ZEA, F.A. (1791), p.210.

19) ZEA, F.A. (1803), El Mercurio de España, Madrid, Tomo I, la Imp. Real, p.7.

20) Estableció especial amistad con A. L. MiLlin, director del Magasin Encyclopédique ou Journal des Sciences, des Lettres et des Arts. En este periódico aparecen noticias sobre distintos botánicos españoles. En especial se incluye a José Antonio Cavanillles, José Celestino Mutis y Francisco Antonio Zea. Véase, Tomo 36, año VI, tomo VI, 1801, pp. 112, 113, 114. También en Soto, D. (1995), «Cavanilles y Zea: una amistad político-científica», Asclepio, vol. XLVII, fasc.1. 
tor de La Gazeta y El Mercurio ${ }^{21}$ de Madrid, refleja su tendencia francesa en las informaciones políticas y científicas que difunde desde estos dos diarios.

El Mercurio, periódico estatal, se componía de dos apartados: el político y el literario. En la sección política, Zea expresó sin reparo su apoyo y admiración hacia el gobierno y científicos franceses. Es significativo que desde el primer número, se declaró admirador de Napoleón y le llegó a considerar el salvador de Francia.

En cuanto a su análisis sobre la política española, centra sus elogios en el Príncipe de la Paz(1767-1847). En Godoy reconoce al gran ministro que ha organizado el ejército, los cosmógrafos del Estado, apoya los progresos de las ciencias y la instrucción pública. El Ministro era, para el criollo, el que impulsaba las ideas liberales y a su alreedor se encontraba el grupo "avanzado" de los afrancesados. Era evidente que Zea se consideraba integrante de este grupo. No es extraño, por tanto, que tomara la posición de defender el trabajo de Godoy que en concreto en los planes educativos se veían entorpecidos por el grupo tradicional ${ }^{22}$. Años más tarde se manifestaría en los motivos del cierre del Instituto Pestalozziano.

Resulta fundamental insistir que Zea, desde esta palestra, se hace portavoz de la política real y de la que lleva a cabo el ministro Godoy. Su mensaje en los citados periódicos es triunfalista. Manifiesta que se está en un país en camino de conseguir el bienestar general y se trabaja por el progreso de las ciencias. Afirma que: «En efecto, nuestro comercio se va restableciendo.... y todo anuncia que se nos acerca una grande época de esplendor y prosperidad» ${ }^{23}$.

El director del periódico no deja de destacar la importancia que tenía América para el desarrollo de la moderna España. El país que vislumbra Zea tiene sus cimientos en una relación apoyada en los intereses de las colonias con los de la metrópoli. La clave estaba en la organización y en el comercio. Según él se debían organizar todos los ramos de rentas y comercio, la justicia y las ciencias, las artes y la agricultura. Por otra parte, el comercio permitía combinar los intereses de «las colonias esencialmente agrícolas con los de la metrópoli esencialmente industriosa» ${ }^{24}$.

En las ciencias, tampoco se quedaba España en la retaguardia. Trató de demostrar que el desarrollo español podía llegar, en un futuro cercano, a compararse con el francés y el alemán. Tal vez por eso procuró dar especial relevancia a la política que impulsaba Godoy. Ya hemos indicado que a este ministro lo señaló con frecuencia, en sus escritos, como el principal impulsor de las ciencias en España. De hecho,

21 Zea fue director desde el 13 de enero de 1803 hasta el 18 de mayo de 1804.

22 El Mercurio de España, enero de 1803, p.38. Carr, R. (1978), España I808 - 1939. Editorial Ariel, Barcelona, $6^{\circ}$ edición, p. 92 .

2.3 El Mercurio, enero de 1803, p.32

24 Ibidem, p.32. 


\section{FRANCISCO ANTONIO ZEA: PERIODISTICA, BOTÁNICO Y POLÍTICO}

indicó que el príncipe de la Paz apoyaba las publicaciones y los trabajos que contribuían a los progresos de la navegación y de las ciencias.

Del mismo modo procuró mostrar que América estaba presente en el desarrollo científico de España. Así, anuncia que: «no tardaría Europa en admirar los grandes e importantes descubrimientos de Mutis, cuyas obras impondrán silencio á los detractores del Ministerio» ${ }^{25}$.

Por los escritos que exponía en El Mercurio da la impresión de que sus proyectos hacia América obedecían a las políticas que señalaba el ministro Godoy. Estos planes se apoyaban en el adelanto de las ciencias, la educación, la agricultura y en el desarrollo del comercio con las plantas americanas. La clave de todo el progreso residía en: «realizar un plan combinado para descubrir las producciones útiles ... en América ... aclimatarlas en España y, luego, estas plantas ya mejoradas y enriquecidas acá y allá en la agricultura se podría comerciar con ellas» ${ }^{26}$.

Otro aspecto que ocupó un lugar destacado, durante el año que dirijió Zea los periódicos de la Gaceta y El Mercurio, fue el de editar mayor información sobre las actividades académicas del Instituto Nacional de Francia. A esta institución estuvo vinculado como alumno en 1801 cuando viajó en comisión oficial por parte del gobierno español ${ }^{27}$.

En este período la temática de los citados periódicos se caracteriza por las noticias científicas de tendencia francesa; una mayor descripción de los libros científicos; una relación de nuevos métodos de enseñanza; la creación de escuelas y actividades de las Sociedades Económicas de Amigos del País. Además, menciona todas las actividades académicas y publicaciones de su protector Cavanilles y da a conocer cuanto libro aparece relacionado con el comercio y la agricultura.

Dentro de los proyectos que anunció el director de El Mercurio está el de la creación de 24 establecimientos botánicos ${ }^{28}$. Una de las finalidades de estos Jardines era el de la aclimatación agrícola de las plantas americanas en España para luego desarrollar su comercio. El objetivo en este caso era formar profesionales en el ramo de la agricultura y en el comercio. Es decir, su antiguo proyecto de formar el «botánico ecónomo». El proyecto se justificó dentro de la política ilustrada de unir «lo útil con la formación científica».

\footnotetext{
25 Ibidem, p.35.

26 Ibidem, pp.31-39.

27 Zea no sólo publicó las actividades académicas del Instituto Nacional de Francia sino que también describe los trabajos de los miembros de este Instituto que aparecen en el Memorial Literario o Biblioteca periódica de Ciencias y Artes. SOTO, D. (1995).

28 Archivo Jardín Botánico (en adelante AJB), ref. I, 22, 4, 12.
} 
Consideramos que el trabajo en estos periódicos le aportó a Zea, sin lugar a dudas, una gran experiencia que, luego, reflejaría en la dirección de El Correo del Orinoco.

ZEA DIVULGADOR DEL PENSAMIENTO BOTÁNICO Y AGRÍCOLA EN LA PRENSA ESPAÑOLA

\footnotetext{
«Por fin ha prevalecido la voz del bien contra los clamores de la vanidad, y en todas partes se va dichosamente estableciendo la alianza natural de la botánica con la agricultura» ${ }^{29}$.
}

Francisco Antonio Zea deja la dirección de los periódicos La Gaceta y El Mercurio cuando pasa a ocupar la dirección del Real Jardín Botánico. En su nuevo cargo pudo desarrollar gran parte de las iniciativas que venía planteando en los citados periódicos, sobre la botánica y la agricultura. En estos planes contó siempre con el apoyo del grupo francófilo y en especial con la aprobación del ministro Godoy y del Secretario de Estado Pedro Cevallos.

Es necesario valorar, en su justa medida, la labor que realizó Zea desde el Semanario de Agricultura y Artes, en la divulgación de la botánica y las experiencias agrícolas nacionales y extranjeras.

Este periódico lo crea Juan Antonio Melón y lo cede, desde 1804, al Real Jardín Botánico de Madrid. El objetivo del Semanario de Agricultura y Artes, que iba dirigido a los párrocos, era «el fomento del desarrollo agrícola y el de aquellas artes y manufacturas próximas al quehacer del agricultor» ${ }^{30}$.

Desde la creación de este Semanario, en 1797, hasta el número de 1808, cuando deja de editarse, se publica un gran número de artículos que propugnan por la creación de instituciones, cátedras, planes de enseñanza para escuelas agrícolas de primeras letras y utilización de nuevos métodos de enseñanza aplicados al ramo de la agricultura.

29 F.A. ZEA, Semanario de Agricultura y Artes, $\mathrm{n}^{\circ}$ 490, 22 de mayo de 1806, p.329.

30) El primer número de este periódico aparece 4 de julio de 1797 y el último el 23 de junio de 1808. La dirección del Semanario la asumen Zea y los profesores del Jardín a partir del 4 de julio de 1805 y publicaron 45 números de los cuales el director del Jardín participó directamente con sus escritos en 38 . AJB, ref. I, 22, 1, 15. 


\section{FRANCISCO ANTONIO ZEA: PERIODISTICA, BOTÁNICO Y POLÍTICO}

Cuando el periódico pasa al Jardín Botánico ${ }^{31}$ se asume en dirección colectiva por parte del director del Jardín y de los profesores del mismo y nuevamente se reglamenta ${ }^{32}$.

Es evidente que el director desarrolla paralelamente una fecunda labor por institucionalizar la enseñanza agrícola en el Jardín y una labor periodística de divulgación de informes, memorias, discursos, cartas, decretos, métodos y traducciones. Como era de esperar permaneció fiel al ideario político-académico francés que había defendido en la Gaceta y El Mercurio. Ahora, El Semanario servía igualmente para difundir la política del ministro Godoy.

La impresión que da Zea es la de una habilidad política puesta al servicio del ministro Godoy y del secretario de Estado Pedro Cevallos. Le concedió especial relevancia a toda la actividad que apoyaron estos funcionarios reales. Ello explica la citación continua que realiza de sus actividades en el campo de la agricultura, la botánica y el desarrollo económico del país. Y, como era de esperar, se presenta un silencio político cuando es apresado Godoy.

En el Semanario, como hemos señalado, el hito importante que le caracterizó fue el de ser un divulgador de la botánica. Sus artículos los hemos clasificado en: discursos; análisis de obras; análisis de experiencias botánicas; y en traducciones de discursos, memorias e informes de extranjeros.

En el apartado de los discursos procuró mostrar su pensamiento de la botánica práctica a través de su aplicación a la agricultura y el comercio. Publicó los discursos que pronunció en la inauguración de las clases del Jardín Botánico de Madrid ${ }^{33}$. Además, editó las Reales Ordenes donde se aprobó sus proyectos bandera que realizó en el Jardín Botánico de Madrid. Estos planes contaron con el beneplácito del ministro Godoy y el secretario Pedro Cevallos. Los proyectos principales fueron: «los 24 establecimientos botánicos con la Escuela Particular»; el Plan de la cátedra de agricultura y el destino de los alumnos de la Escuela de Agricultura ${ }^{34}$.

El fue consciente de la importancia que suponía dar a conocer los escritos botánicos en un órgano de difusión como era el Semanario. Sus palabras son muy claras en el análisis de obras botánicas cuando dice que: «una de las grandes utilidades de los papeles periódicos es dar a conocer obras dignas de la atención pública, analizarlas y hacer formar concepto de su mérito e importancia» ${ }^{35}$. Este objetivo fue el que se

31 Debido a las discrepancias entre Zea y Melón el periódico sufre varias reglamentaciones. AJB, ref. I, 22, 1, 22. AJB, ref. I, 22, 3, 15. AJB, ref. I, 22, 5, 7 .

32 AJB, ref. I, 22, 6, 3. AJB, ref.I, 22, 6, 6. AJB, ref. I, 22, 6, 7 .

33 El discurso de 1805 se publicó en los números 458 y 460 . El discurso de 1806 se editó en los números 461,462 y 463

34 Véase $n^{\circ} 589$ del 14 de abril de 1808.

35 Véase $n^{\circ} 464$ del 21 de noviembre de 1805. 
propuso cuando reseñó la obra de sus amigos Claudio y Esteban Boutelou, Extracto del tratado de las flores.

Como era de esperar, en cada presentación no pierde la oportunidad de dar a conocer su pensamiento sobre la importancia de la agricultura para el desarrollo de un país. No es por tanto extraño encontrar en «Advertencia» ${ }^{36}$ sobre el escrito Ensayo sobre las variedades de la vid común de Simón de Roxas Clemente, y sobre el cultivo de esta planta por Esteban Boutelou, una especial justificación que años atrás había expuesto en El Mercurio de España sobre el desarrollo agrícola.

Resulta interesante destacar en su análisis de las experiencias botánicas la vinculación que establece entre ciencia y política. Vuelve a insistir por tanto en el apoyo que el ministro Godoy le da a estas actividades. Es significativo el título que le da a «Reflexiones sobre los adelantamientos .. de la Real Sociedad Patriótica de San Lúcar, desde que nombró por su director al Señor Príncipe de la Paz» ${ }^{37}$. En este artículo además de resaltar el apoyo del Príncipe de la Paz a la institución desarrolla sus argumentos sobre los beneficios que trae a la sociedad el hacer populares los conocimientos útiles de las artes y la agricultura. Igualmente trata de explicar la importancia de los estudios sobre los recursos del país que analizan el estado de la industria rural. No deja de ser curioso que en este escrito reclame para sí el honor de haber introducido en España la vacuna que descubrió Jenner ${ }^{38}$.

$\mathrm{Al}$ año siguiente, vuelve a insistir en el análisis sobre el Jardín de San Lúcar con el motivo de la celebración del aniversario de la Real Sociedad Económica de San Lúcar de Barrameda. Como era de esperar repite los criterios que sobre esta experiencia ya había señalado en relación al ministro y a la importancia de la agricultura para el desarrollo del país ${ }^{39}$. El mérito de la idea de la fiesta se lo concede a Francisco Amorós.

36 Véase $\mathrm{n}^{\circ} 522$ del 1 de enero de 1807

37 Véase el número 471 del 9 de enero de 1806.

38 Dice Zea: «Aunque en casi todas las Gazetas de Europa se anunció á su tiempo que un amigo mio y yo la habíamos introducido en España, creo necesario manifestarlo como lo haré en otro número. $\mathrm{Ni}$ uno ni otro hemos solicitado por tan importante servicio recompensa ni premio alguno; lo hicimos por amor a la humanidad, tuvimos mucho que trabajar porque era entonces problemática su utilidad, y semejantes descubrimientos no han de propagarse á la buena suerte; y por lo mismo no queremos que se nos defraude de la gloria á que tenemos derecho». El Semanario, nº 471, p. 19.

39 En esta ocasión reseñó la «Noticia de la fiesta primera con que la Real Sociedad Económica de San Lúcar de Barrameda celebró en el 1 de enero de este año la fundación del Real Jardín experimental y de aclimatación y el nombre de su ilustre protector el Serenísimo Sr. Príncipe Generalísimo Almirant». Véase los números 532 del 12 de marzo de 1807, n 534 del 26 de marzo de 1807, n 535 del 2 de abril de 1807. 
Dentro de la misma perpectiva de establecer la relación entre el desarrollo agrícola y la prosperidad de las fabricas reseña la «Noticia del arte de amoldar las maderas finas para copiar todo género de decoraciones, obras de talla y aun estatuas» ${ }^{40}$.

Resulta fundamental insistir en cómo vinculó su habilidad política en los escritos científicos. En el articulo «Noticia de la expedición encargada de propagar la vacuna en ambas Indias, y del aprecio que ha hecho a Europa de la filantropía de nuestro gobierno», refleja el cambio político que se estaba viviendo en 1808 en España ${ }^{41}$. En este año crítico de 1808, la posición del periódico fue la de no mencionar al Príncipe de la Paz y su alabanza se inclinó hacia el Rey de España y Napoleón. Es bien conocido que Zea no fue partidario de Fernando VII. Por el contrario eran públicas sus manifestaciones de afecto hacia Napoleón. En esta época el criollo no pregonó la independencia de las colonias americanas. Su pensamiento político ya era conocido cuando había expresado en la Gaceta y El Mercurio la necesidad de un trato de igualdad entre las colonias y la metrópoli. Este objetivo estaba acompañado de su creencia en un Napoleón que era capaz de conseguir el progreso del país ${ }^{42}$.

Las notas e introducciones que realizó a las 27 traducciones $^{43}$ que hemos registrado, son interesantes. Su hilo conductor fue invariable. Con gran frecuencia unió en sus escritos a los personajes de la política y de la ciencia.

No es extraño, por tanto, encontrar en la traducción sobre el «Discurso de Mr. Parmentier» ${ }^{44}$ la alabanza que realiza el científico al Príncipe de la Paz por haber establecido en Madrid la Escuela de Veterinaria.

Es evidente que enfoca siempre sus análisis a destacar al grupo afrancesado y a reseñar la importancia de la agricultura en su preocupación de hacerla «apreciar» por el público ${ }^{45}$. Dentro de este panorama tradujo el discurso de M. Chassiron, en la Junta pública de la Sociedad de Agricultura de París.

Es necesario valorar su aporte en las traducciones de Memorias por la variedad de temas que da a conocer. La mayoría de éstos se relacionan con la agricultura, la alimentación, creación de escuelas, otras sobre la industria y la veterinaria. Algunos

40) Véase no 560 del 24 de septiembre de 1807.

41 Recordemos que 1808 es el año de la caída de Godoy. El 17 de marzo se le destituye; el 29 de marzo se le embargan los bienes; el 2 de mayo, en el levantamiento de Madrid, se le saquea la casa. Carr, R. (1978), pp. 4, 89, 93. Príncipe de la Paz, Memorias (edición y estudio preliminar de Carlos Seco Serrano), Biblioteca de Autores Españoles, Madrid, 1965.

42 CARr, R. (1978), p.93. Semanario, nº 580 del 11 de febrero de 1808.

43 Estos escritos los hemos clasificado en: discursos, memorias, informes y cartas.

44 «Discurso que Mr. Parmentier pronunció en la Junta de la Escuela Imperial de Veterinaria de Alfort en el Departamento del Sena para la distribución de premios». Véase $n^{\circ}$ s 465 del 28 de noviembre de 1805 y 466 del 5 de diciembre de 1805.

45 Semanario de Agricultura y Artes, números 493 del 12 de junio de 1806; 494 del 19 de junio de 1806 y 495 del 26 de junio de 1806. 
escritos establecen conexión entre el desarrollo económico de Inglaterra y de Francia. En este trabajo sólo relacionaremos algunas traduciones que tienen vínculo con la agricultura.

Fue manifiesto su interés por publicar las experiencias educativas que tuviesen que ver con la enseñanza agrícola. En esta línea dio a conocer la traducción que realizó Claudio Boutelou sobre «el establecimiento de una escuela de árboles frutales en el Jardín Botánico de París» ${ }^{46}$. La más importante novedad en esta «Adición» es la crítica que realiza a los Jardines Botánicos. Sus palabras son muy claras al señalar que «No se puede negar que los Jardines Botánicos han sido hasta ahora establecimientos de luxo: que lo era la ciencia misma, y que el público tenía razón en preguntar constantemente para que servía, no viendola producir ningún efecto manifiestamente ventajoso ${ }^{47}$. En este caso, en su esfuerzo por ser práctico, considera que en estos Jardines se deben introducir plantas útiles y destinarlos a la enseñanza como un medio para restablecer la alianza natural de la Botánica con la agricultura.

Del mismo modo trató de dar a conocer memorias sobre el estado agrícola en España y experiencias concretas de este ramo. Podemos destacar la que Tessier ${ }^{48}$ había presentado en la Academia de Ciencias de París sobre «el estado de la agricultura en las Islas Canarias» ${ }^{49}$. En el mismo número del Semanario traduce el «Extracto de una memoria de Mr. Giobert sobre los abonos» que estaba inserta en el quinto volumen de la obra intitulada Memoria della R. Societá agraria, en $1790^{50}$.

Hemos comentado que el referente, casi habitual en Zea, son las publicaciones científicas francesas. Quizá una de las más nombradas sea los Annales des Arts et Manufactures. De estos Annales traduce el «Extracto de una memoria sobre las causas de la prosperidad de las manufacturas inglesas en contraposición de las francesas» ${ }^{51}$

No deja de ser curiosa la traducción que realiza de Mr. Toulongeon sobre el «Influxo del régimen dietético de una nación en su estado político» que leyó en el Instituto de las Ciencias de París ${ }^{52}$. En la nota habitual de sus traducciones, señala

46 «La Escuela de árboles frutales que estableció en París Monsieur Thouin». Se publica en los números 488 y 490 . Thouin era el jardinero mayor de París y fue uno de las personas que contacto Zea en París por intermedio de Cavanilles.

47 El Semanario de Agricultura y Artes, $\mathrm{n}^{\circ} 488$, p. 328.

48 De Tessier también traduce «Memoria sobre los abusos de los rompimientos», que había leído en el Instituto de Ciencia. En este mismo número, 469 del 26 de diciembre de 1805, traduce Zea la «Memoria sobre las lanas de Chachemira, cria y cuidado de carneros y fábricas de los chales», de Mr. Legoux de Flaix, oficial retirado del Cuerpo de Ingenieros.

49) Se publica en los números 467 y 468 .

50) Publica la traducción en los números 467 y 468.

51 Véase Semanario n ${ }^{\circ} 557$ del 3 de septiembre de 1807.

52 Este artículo se publicó en el Semanario número 475 del 6 de febrero de 1806. 


\section{FRANCISCO ANTONIO ZEA: PERIODISTICA, BOTÁNICO Y POLÍTICO}

aquí «que la memoria es digna de la mayor atención» y que en otro lugar manifestará el objeto del porqué la ha traducido y las consecuencias que deduce de ella.

En las introducciones que realiza para los informes, ocupa un lugar destacado la medicina botánica que contribuye a la veterinaria y a la perfección de la agricultura. Tal vez por eso, orientó sus traducciones a escritos como el «Extracto de un informe a la Sociedad de Agricultura del departamento del Sena y del Oisa, dado por Mr. Voisen á nombre de una comisión especial acerca de la vacunación y de las viruelas del ganado lanar» ${ }^{53}$. Este escrito es otro de los tantos que iban dirigidos por los autores al Príncipe de la Paz. En la introducción que realiza a este artículo vuelve a recordar el honor que le corresponde por haber introducido la vacuna en España.

\section{ZEA DIVULGADOR DEL MÉTODO DE PESTALOZZI EN ESPAÑA}

\footnotetext{
«Mi ideal de la educación de esos niños comprendía la agricultura, la industria y el comercio. Yo poseía en esos tres ramos un elevado y seguro tacto para el todo y lo esencial de ese plan, y aún hoy mismo no veo ningún error en los fundamentos de él» ${ }^{54}$.
}

Por ser de especial interés la aplicación del método pestalozziano en España dedicamos un apartado al análisis del mismo. Además, hasta ahora no se ha valorado la participación que tuvo Francisco Antonio Zea, desde la dirección del Real Jardín Botánico, en la divulgación de este método.

Resulta interesante encontrar cómo el criollo desde su regreso de París, en 1802, se une al equipo de Godoy a través de Cavanilles. Este grupo consideró prioritario la enseñanza del pueblo para conseguir la prosperidad del país. De hecho, en esta época se despertó la curiosidad por los nuevos métodos de enseñanza que se practicaban en el extranjero. Ello explica su compromiso desde la Gaceta y El Mercurio de dar a conocer los nuevos métodos que se experimentaban en otros países.

De todas formas es importante apuntar que la aplicación y la cancelación de la experiencia metodológica pestalozziana está íntimamente ligada al ministro Godoy. En su momento la aplicación de este método se convirtió en una de las prioridades del Príncipe de la Paz. Se trabajaba en libros para enseñar «la religión, la historia, la

53 Semanario de Agricultura y Artes, números 478, 479 y 481.

54 Johann Heinrich Pestalozzi nació en 1746 en Zurich, Suiza, y murió en Brugg en 1827. Entre sus obras más divulgadas en España están: Cómo Gertrudis enseña a sus hijos (1801) y El manual de madres. La primera obra la tradujo Juan O. Monasterios, LEIPZIG, P.A. Brookaus, 1808, 278 págs. Nosotros hemos consultado la traducción de José Tadeo Sepúlveda, con prólogo y notas de Ricardo Nervi, Buenos Aires, Centro Editor de América Latina, S.A., 1967, p.42. 


\section{DIANA E. SOTO ARANGO}

moral, las leyes patrias, la economía política, los preceptos higiénicos» ${ }^{55}$. El impacto del Instituto Pestalozziano trajo consigo la división de opiniones. El sector opositor a Godoy combatió esta institución por identificarla como un ataque a la religión y al dogma cristiano.

Como puede verse el director del Real Jardín Botánico de Madrid, a través del Semanario de Agricultura y Artes, se unió al grupo que divulgó el método pestalozziano por petición expresa de Pedro Cevallos ${ }^{56}$. A los pocos días edita el primer artículo titulado «Idea del nuevo método de enseñanza de Henrique Pestalozzi». Este escrito lo realiza el clérigo Juan Andújar ${ }^{57}$, secretario del Duque de Frías y redactor de la Gaceta de Madrid, quien tradujo en España varias obras de Pestalozzi.

Los manuscritos que Andújar hizo traducir a su costa y redactó la traducción fueron Cómo Gertrudis enseña a sus hijos, El libro de las madres, Los libros elementales. En realidad el escrito que se publica en el Semanario, como artículo elaborado por Andújar, es un resumen de la traducción de Alex Chanes de $1807^{58}$. El escrito de Andújar lo consultó Rufino Blanco Sánchez en el Archivo General Central de Alcalá, en 1909. Los documentos ya no existen porque se quemaron en el incendio del Palacio Arzobispal, en 1939, que era el sitio donde funcionaba el citado Archivo. No

55 Señala Godoy en la p. 135 de sus Memorias que el presbítero don Juan de Andújar, uno de los literatos de la comisión que fue nombrada, había ya traducido cinco obras elementales de Enrique Pestalozzi, e hizo presente de ellas por mi mano al Instituto.

56 AJB, ref.I, 22, 6, 20. «El nuevo método pestalozziano» se publicó en el Semanario de Agricultura y Artes en los números 505, 506 y 507 del 4, 11 y 18 de septiembre de 1806.

$57 \mathrm{El}$ artículo que publicó Zea se editó al año siguiente en Noticia de las Providencias tomadas por el gobierno para observar el nuevo método de enseñanza primaria de Enrique Pestalozzi, y de los progresos que ha hecho el establecimiento formado en Madrid con este objeto, desde su origen hasta principio del año 1807, Imp. Real, Madrid, 1807. Este documento bajo el título «Idea del nuevo método de enseñanza de Henrique Pestalozzi» lo consultó Rufino Blanco en el Archivo de Alcalá, quien señala que también se publicó en la Habana, en 1807. BLANCo SÁnCHEZ, R. (1909), Vida y obra de Pestalozzi. Pestalozzi en España, Imp. Revista de Archivos, Madrid. Morf, H. (1928), Pestalozzi en España, Museo Pedagógico, Madrid. En 1887 se publicaron estos trabajos en español. Aparecieron en el Paedagogium, Revista de Viena y fueron traducidos en el número de enero y siguientes de 1887 en el Boletín de la Institución Libre de Enseñanza, t.X, 1887, pp. 20-22, 52-54, 115-123. Sureda García, B. (1985), «Los inicios de la difusión del método de Pestalozzi en España. El papel de los diplomáticos españoles en Suiza y de la prensa periódica», Revista de Historia de la Educación. Revista Interuniversitaria, $\mathrm{n}^{\circ} 4$, enero-diciembre, pp.35-62.

58 Chavannes, A. (1807), Exposición del método elemental de Henrique Pestalozzi, con una noticia de este célebre hombre, de su establecimiento de educación y de sus principales cooperadores. Traducida al castellano por Don..., Gómez Fuentenebro, Madrid. En este mismo año publica BLANCO CRESPO, J.M. (1807), Discurso sobre ¿si el método de enseñanza de Enrique Pestalozzi puede apagar el genio, y especialmente el que se requiere para las artes de imitación?, Gómez Fuentenebro, Madrid. 
sabemos si estas obras ${ }^{59}$ se llegaron a publicar porque el mismo Godoy comenta que «No estoy bien cierto si se llegó a imprimir aquella obra Cómo Gertrudis enseña a sus hijos, pero el manuscrito fué enviado a la Imprenta Real, mandándose darla a luz pública con preferencia a otras muchas que estaban encomendadas» ${ }^{60}$.

En el citado artículo del Semanario puede verse cómo Zea insiste en las dos ideas que marcan sus comentarios. La primera en torno al apoyo e impulso que da a la enseñanza agrícola en las escuelas, y la segunda en el reconocimiento público a la actividad que realizaba el ministro Godoy.

Por las razones ya argumentadas apoya el método pestalozziano porque considera que se aplica «con mucho acierto a la enseñanza agrícola» ${ }^{61}$. Por otra parte, y más exactamente en la tercera entrega, de los artículos señalados, escribe sobre las ideas liberales ponderando al Príncipe de la Paz por tener este pensamiento ${ }^{62}$.

Paralelamente a las publicaciones, se fue organizando el reglamento para el gobierno de la escuela pestalozziana de Madrid ${ }^{63}$. Resulta fundamental insistir que el Príncipe de la Paz impulsó personalmente la creación de esta escuela y subvencionó parte de sus gastos, porque «no alcanzaban los medios del Estado destinados a este objeto» ${ }^{4}$. La «Escuela Central y Normal del Instituto Real Pestalozziano fue abierta con gran solemnidad, en las Casas Consistoriales de Madrid, el 4 de noviembre de 1806». Esta noticia fue ampliamente comentada en los periódicos madrileños ${ }^{65}$.

Esta institución funcionó hasta el 13 de enero de 1808 cuando el ministro Godoy, su protector, ordena la clausura del Instituto Pestalozziano y en la misma fecha se cierra también el de Cantabria. En la carta que Godoy le envía a Pestalozzi le comu-

59 Parece que de Andújar lo que se publicó oficialmente fue el escrito que se editó en el Semanario (1806) y en la Imprenta Real (1807). Juan Andújar en 1807 publicó El ABC de la visión intuitiva o principios de la visión relativamente a los tamaños. Parte primera, Imp. Real, Madrid, 1807, 302 pp.

61) Godoy (1965), p.138. Según Rufino Blanco estas obras de Pestalozzi que hizo traducir Andújar no se llegaron a publicar en la Imprenta Real porque el Príncipe de la Paz dio la Orden de suspender estas ediciones el 13 de enero de 1808, BLANCO SÁNCHEZ R. (1909), p.471.

${ }^{6} 1$ Semanario de Agricultura y Artes dirigido a los párrocos por el Real Jardín Botánico de Madrid, t.XX, Imp. de Villalpando, $\mathrm{n}^{\circ}$ 505, pp. 145-153.

${ }_{62}$ Zea publica bajo el título «Concluye el nuevo método de enseñanza de Henrique Pestalozzi. Aplicación al estudio de la geografía», en Semanario de Agricultura y Artes, nº 507, pp. 181-192. Notas de Zea en pp. 186, 188, 192.

6.3 Archivo Sociedad Económica de Amigos del País (en adelante ASEAPM), legajo 199, doc.33. ASEAPM, legajo 199, doc.34.

64 Godoy (1965), Memorias, p.138.

65 La Gaceta de Madrid, del viernes 7 de noviembre de 1806. El Mercurio de Madrid del 30 de noviembre de 1806. La crónica que aparece en este periódico es similar a la de La Gaceta aunque un poco más extensa. Godoy, en las citadas Memorias, describe la inauguración así: «la Escuela comenzó con un gran número de niños, con unos treinta seis maestros de primeras letras y con los cien discípulos observadores, cuyo modesto título eligieron las personas recibidas de Madrid y las Provincias», p.138. 
nica que ha tenido que cerrar el Instituto por «la ingratitud de unos y la ignorancia de otros». Se disculpa por su decisión tomada a pesar de estar de acuerdo con el método y como prueba le comenta a Pestalozzi que «educará por el sistema pestalozziano a 12 huérfanos militares» ${ }^{66}$.

Pero «no todo pereció en la borrasca de 1808», porque el secretario de la Institución, Francisco Amorós, retoma la experiencia española y, en Francia, organiza el Gimnasio Normal parisiense. Amorós, además, fue «inspector general de los demás Gimnasios militares» de Francia. Godoy comenta: «¿Quien me habría dicho a mí, cuando se comenzaba en España esta grande obra, que otra nación más dichosa sería la que sacaría fruto de ella, y que la misma tierra que era el centro de las luces y que tenía sobrado de su propio fondo para repartir y dar en todas partes, aceptaría y se haría propia suya, no tan sólo una parte de esta obra, sino también el mismo obrero» ${ }^{67}$.

En síntesis, la aplicación del método se acompañó de una amplia difusión de los adelantos de los estudiantes y de una síntesis de los principios de la enseñanza pestalozzianos. La relación de Zea con el método pestalozziano se da a través de la importancia que le da a este método para la enseñanza de la agricultura en las escuelas. En este contexto, y por su incondicional apoyo a las actividades del Príncipe de la Paz, divulga el método de enseñanza agrícola en el Semanario de Agricultura y Artes.

\title{
EL CORREO DEL ORINOCO: UNA EXPERIENCIA POLÍTICO-PERIODÍSTICA PARA ZEA
}

\begin{abstract}
«Yo creré haber hecho un servicio a mi patria y a la humanidad, si presentando al público estos apuntamientos, logro que algún sabio filántropo se levante indignado contra la tiranía, tome a su cargo la defensa de los pueblos oprimidos y haga ver a los gabinetes ilustrados la justicia y la necesidad de contener el furor de un gobierno antropófago, dos veces desolador de un continente inmenso» ${ }^{68}$.
\end{abstract}

Resulta interesante encontrar, unos años después, al criollo Francisco Antonio Zea en su papel de dirigente político revolucionario. El centro de operaciones políticas fue la ciudad de Angostura. Desde esta población fundó y orientó el Correo del Orinoco $^{69}$.

66 MORF, H. (1928), p.48.

67 Godoy (1965), p.139.

68 F. Zea, El Correo del Orinoco, n 15,21 de noviembre de 1818.

69 Este periódico publica el primer número el sábado 27 de junio de 1818 y edita el último número el sábado 23 de marzo de 1823. El editor del periódico fue Andrés Roderick, quien se intítulo «Impresor del Supremo Gobierno». La imprenta se instaló en la casa $n^{\circ} 83$, de la Calle de La Muralla, en la ciudad 
$\mathrm{Al}$ movimiento revolucionario de la independencia se vincula por medio de Miranda. Parece ser que el grupo de la masonería francesa a la que pertenecían Bolívar y Miranda ${ }^{70}$, y posiblemente también Zea, apoyará la gesta libertaria de las colonias americanas.

Para entender la posición política de Zea, desde la dirección del periódico Correo del Orinoco, se debe recordar que el criollo no fue partidario de Fernando VII. Por el contrario, por su comportamiento de apoyo a Godoy y posteriormente a José I de España fue condenado a muerte. La base de esta condena, en 1813, fue el de ser traidor a la causa de Fernando VII. Esta situación le llevó a refugiarse primero en París y luego en Londres con su mujer y su hija ${ }^{71}$.

Conviene destacar que el impacto que estaba cobrando el movimiento de la independencia americana en Europa y su amistad con Miranda no fue lo único que le llevó a trasladarse a América. Consideramos que mediaban intereses más fuertes, como eran posiblemente los de la masonería que en su relación más o menos direc$\mathrm{ta}^{72}$, le conducen a viajar, en la primavera de 1815 , a la isla de Santo Domingo donde conoce a Bolívar. Es importante apuntar que desde esta fecha se establece una estrecha amistad con el «libertador». De hecho, se convertiría más adelante en uno de sus más fieles consejeros en la elaboración del perfil que se le daría a la Constitución de la denominada Gran Colombia y como vice-presidente de esta naciente República.

Tampoco hay que olvidar la claridad del pensamiento político que el criollo fue elaborando en su tendencia francófila desde las tertulias de Santa Fe con Nariño. Como puede verse, para esta época su objetivo era trabajar por la independencia americana. Esto explica su rechazo a la dirección del ramo de la Agricultura que le hizo el presidente de la República de Haíti ${ }^{73}$. Lo imporante era vincularse al grupo

\footnotetext{
de Angostura, Venezuela. Se consultó la reproducción facsimilar que se publicó en conmemoración del Cincuentenario de la Academia Nacional de Historia, Desclée de Brouwer, \&. A., Paris, 1939.

70) La vinculación masónica de Bolívar y Miranda ha sido puesta en tela de juicio por varios historiadores. En especial, el caso de Bolívar por su decreto del 8 de noviembre de 1828 que condena la masonería. Por el contrario, Americo Carnicelli defiende la vinculación masónica de estos dirigentes revolucionarios. Este historiador publica la inscripción de Bolívar en la Masonería «San Andrés de Escocia», de París. El mismo autor afirma que Zea era masón pero no aporta la respectiva documentación. Véase Carnicelli, A. (1970), La masonería en la independencia de América, Cooperativa Nacional de Artes Gráficas, Bogotá, t.II, p.350. También, Navarro, N.E. (1928), La masonería y la independencia a propósito de unos "reparillos". Ofrenda a la memoria de Bolívar en el año centésimo de su inmortal decreto de 8 de noviembre de 1828 condenatorio de la masonería, Ed. Sudamericana. Caracas; Junco, A. (1952), «La masonería condenada por los prohombres de la independencia», Cucadernos Hispanoamericanos, $\mathrm{n}^{\circ} 30$, pp.205-303.

71 Botero (1945), p.91.

72 Tenemos la información da la vinculación masónica de Carlos Molina y José María Lanz a quienes contrató Zea en 1822 .

7.3 Botero (1945), p.94.
} 
de dirección del movimiento de la independencia y por este motivo se traslada a Venezuela.

Una vez instalado en la ciudad de Angostura su pasión periodística le lleva a fundar, como ya hemos señalado, el Correo del Orinoco. Este medio se convirtió en el órgano oficial del nuevo gobierno que aún luchaba por la independencia de su territorio. El periódico salía el sábado de cada semana y se organizó en cuatro apartados. 1. Decretos y Actas del Gobierno; 2. Noticias comerciales; 3. Noticias de periódicos extranjeros; 4. Variedades.

El carácter de esta publicación fue eminentemente político; por lo tanto, el apartado de información científica que había cultivado Zea en el Semanario de Agricultura y Artes, la Gaceta y El Mercurio de Madrid, se vió relegado sólo a noticias ocasionales. La pluma sarcástica del criollo vuelve a la palestra contra sus oponentes, bajo la única prioridad de la independencia de América, donde se puede analizar su pensamiento político.

El trabajo político de Zea no fue en el campo de batalla. Su habilidad política, facilidad de expresión y en especial el posible vínculo masónico le llevan a ocupar el lugar de consejero de Bolívar ${ }^{74}$ y a desempeñar cargos en la dirección política del nuevo gobierno. Estos puestos fueron entre otros: Intendente (12 de febrero de 1816); Miembro de la Junta Provisional del gobierno (8 de mayo de 1817); Presidente del Tribunal de secuestro de los bienes de los partidarios del rey (23 de septiembre de 1817); Presidente de «Estado y de Hacienda» (10 de noviembre de 1817); Diputado ante el Congreso (febrero de 1819); Presidente del Congreso (15 de febrero de 1819); Vice-presidente de la República (15 de febrero y 24 de diciembre de 1819) y finalmente Ministro Plenipotenciario en el Exterior( 24 de diciembre de 1819).

El Correo del Orinoco estaba destinado a la élite criolla neogranadina. Se escribían algunas referencias en francés y se llegó a publicar algún artículo en inglés ${ }^{75}$.

En sus escritos pronto identificó al principal enemigo que se tenía en tierras americanas procedente de España. Su ironía la enfiló contra este personaje que se autodenominaba «el pacificador Morillo». En su sentimiento nacionalista y de respeto a la ciencia no le perdonó a Morillo el haber sacrificado a los hombres sabios de su tierra $^{76}$.

\footnotetext{
74 Se debe recordar que en esta época Bolívar era masón. El rompimiento con los masones se da posiblemente a partir de septiembre de 1827

75 Véase «Entrance and navigation of the Orinoco», publicado en el $n^{\circ} 7$, del sábado 8 de agosto de 1818.

76 El Correo del Orinoco, $\mathrm{n}^{\circ}$ 13, sábado 17 de octubre de 1881.
} 
Dentro de la general relevancia que concedemos a sus escritos políticos en este periódico, ocupa un lugar destacado «La Mediación entre España y América» ${ }^{77}$. Este texto se lo encomendó Bolívar para que se debatiera la solicitud de intervención de las cortes europeas en los destinos de América.

Un hito importante del artículo es la diferencia que estableció entre el gobierno de Fernando VII y los que le precedieron, como el de Carlos IV, donde él participó en la dirección del Real Jardín Botánico de Madrid. Sus palabras son claras cuando señala que: «Mucho antes que la América se resolviese a esta declaración augusta, no faltaron españoles sabios y celosos, que se esforzaron vanamente en apartar del precipicio a su demente gobierno» ${ }^{78}$. Como es de suponer no faltó en este artículo sus menciones a la libertad de comercio, el desarrollo agrícola y, en especial, el recuerdo a los científicos europeos como Jussieu, Cuvier, Vauquelin, entre otros.

El impacto del documento no se dio sólo por la lectura que se hizó a través del Correo del Orinoco. La especial repercusión se da en la lectura que se hizo del «Manifiesto» en el Congreso, el 20 de enero de 1820, y en el trámite oficial del mismo en Europa.

Aunque el momento era de intrigas políticas en la cúpula del gobierno revolucionario, a Zea se le reconoce su habilidad de diplomático y se le envía a Europa con el cargo de «Vicepresidente de la República de Colombia y Enviado Extraordinario y Ministro Plenipotenciario ante los gobiernos de E.U. de América y varias cortes europeas» ${ }^{79}$. En esta misión podría firmar empréstitos, llegar a acuerdos políticos y contratar personas que viniesen a servir al país.

Es dentro de este cometido donde su documento la «Mediación» va a tener las repercusiones políticas esperadas. En esta ocasión el escrito extenso lo reduciría a términos más diplomáticos, en la nueva versión que pasó oficialmente por carta al Duque de Frías ${ }^{80}$ en octubre de 1820.

77 El escrito «La Mediación» se publica en varias entregas. En 1818 las realizó en: $n^{\circ} 7,8$ de agosto; $\mathrm{n}^{\circ} 8,15$ de agosto; $\mathrm{n}^{\circ} 10,29$ de agosto; $\mathrm{n}^{\circ} 11,5$ de septiembre; $\mathrm{n}^{\circ} 15,21$ de noviembre; En 1819 continúo publicando el artículo en el $n^{\circ} 17,6$ de febrero; $n^{\circ} 18,13$ de febrero; y el último en el $n^{\circ} 19$ del 20 de febrero.

78 El Correo del Orinoco, ${ }^{\circ} 10,29$ de agosto de 1818.

79 El 24 de diciembre de 1819, Zea es nombrado por Bolívar vicepresidente de la República de Colombia y Enviado Extraordinario y Ministro Plenipotenciario ante los gobiernos de E.U. de América y varias cortes europeas. En la época de la misión diplomática de Zea la República de Colombia la conformaban Venezuela, Ecuador y Nueva Granada. Esta situación geopolítica se mantuvo desde 1818 a 1830. A Zea se le limita su función diplomática sólo a España y se le pide que vuelva al país el 15 de octubre de 1821 .

80 Carta de Zea del 7 de octubre de 1820. AHN, leg.5471, 20 folios. Carta del Duque de Frias a Zea acusando recibo del Plan de reconciliación, Londres, 9 de octubre de 1820, AHN, leg.5471, 2 folios. 
Es necesario, también, valorar otros artículos de carácter político que publicó en el citado periódico. Es evidente que el tema fundamental es la gesta revolucionaria. No es extraño, por tanto, que haga llamadas a la población, y en especial a las mujeres, para que se unan al movimiento, como puede verse en el «Manifiesto» que leyó en el Congreso el día 15 de enero de 1820. Al final de él, realiza una llamada a las mujeres de Colombia. En este texto recuerda a la «inmortal Zalabarrieta» en su liderazgo de heroína americana y señala también otros rumbos a las mujeres en sus misiones de donar «sus joyas a la patria» o en la de «armar a sus hijos contra los españoles» ${ }^{81}$.

Resulta interesante su escrito sobre las «Relaciones de la América del Norte con la del Sur». En este artículo pone de manifiesto el interés de Estados Unidos por la independencia de América del Sur. Es evidente que la posición bolivariana se refleja en este texto. Dentro de esta perspectiva justifica su análisis de la unidad política con Norteamérica. El documento gira en torno a dos ideas: analiza las misiones diplomáticas que había nombrado Estados Unidos hacia los gobiernos independientes del Sur, y manifiesta que América del Norte es republicana y cristiana: por lo tanto apoya la independencia americana. Su pensamiento es claro al expresar que: «son Repúblicanos, y desean el establecimiento de Repúblicas sobre las ruinas del despotísmo, y por que son filántropos y verdaderos cristianos que anhelan la felicidad y la regeneración del hombre oprimido y degradado» ${ }^{82}$. Términa su artículo haciendo una llamada revolucionaria para «arrojar a los Godos» del territorio americano.

Por último queremos destacar que Zea, una vez más, compartió posiciones académicas y políticas con su actividad de redactor en periódicos. Como puede verse, en esta época ejerció el periodismo y a su vez cargos de alta responsabilidad política. No es extraño, por lo tanto, que paralelamente ocupara el cargo de vicepresidente de la República y continuara colaborando en el Correo del Orinoco aunque oficialmente anunció su retirada de la dirección de éste en el número 12. Hay que añadir que este periódico marcó el final de su faceta de periodista y reseñó el final de su vida política en Europa.

\section{CONCLUSIÓN}

Quizá sea la actividad de periodista la que mejor refleje el pensamiento político de Zea y su actividad como divulgador de la botánica. A través de la prensa, se inició como crítico del sistema educativo imperante en la América colonial y a través de este medio se puso al servicio del grupo revolucionario americano.

81 El Correo del Orinoco, $n^{\circ} 50$, sábado 29 de enero de 1820

82 Correo del Orinoco, $\mathrm{n}^{\circ}$ 6, sábado 1 de agosto de 1818. 
El primer contacto con las ideas políticas de Francia lo tuvo a través de la tertulia el «Arcano de la Filantropía» que dirigía Antonio Nariño en Santa Fe. Su participación en la citada tertulia le costó su expatriación que le llevaría a conocer la amada Francia, para él «centro de las ciencias y la libertad».

La estancia en la capital del país galo le sirvió no sólo para su formación académica sino también para la política. Como era de esperar las amistades que estableció en París incidieron en su concepción americanista y científica sobre la botánica.

El criollo pronto se declaró francófilo y así lo manifestó en la dirección de los periódicos de la Gaceta y El Mercurio en Madrid. Por lo tanto, no puede extrañar su firma en la Constitución de Bayona y sus cargos políticos que ocupó durante el corto reinado de José I en España.

En la dirección colectiva del Semanario de Agricultura y Artes desarrolló un intensa actividad en la divulgación de la botánica en España. Su labor más fecunda se centró en el apartado de las traducciones. En este periódico escribió en 35 números y publicó 27 artículos franceses.

El Correo del Orinoco puede considerarse como la culminación de su profesión de periodista. En este órgano escribió uno de los principales documentos políticos sobre la relación que debería darse entre América y España. Este escrito lo tituló «Mediación entre España y América». La entrega oficial del citado documento a España, le llevó a un nuevo encuentro con la denominada «madre patria», a la que amó y atacó con su pluma ágil e irónica de periodista americano. 